\title{
Finite Element Analysis of V-Bending of Polypropylene Using Hydrostatic-Pressure-Dependent Plastic Constitutive Equation*
}

\author{
Kunio Hayakawa ${ }^{1}$, Yukio Sanomura ${ }^{2}$, Mamoru Mizuno ${ }^{3}$, \\ Yukio Kasuga ${ }^{2}$ and Tamotsu Nakamura ${ }^{1}$ \\ ${ }^{1}$ Department of Mechanical Engineering, Shizuoka University, Hamamatsu 432-8561, Japan \\ ${ }^{2}$ Department of Mechanical Engineering, Tamagawa University, Tokyo 194-8610, Japan \\ ${ }^{3}$ Department of Machine Intelligence and Systems Engineering, Akita Prefectural University, \\ Yuri-Honjo 015-0055, Japan
}

In the present paper, V-bending of polypropylene (PP) is analyzed by the finite element method using a plastic constitutive equation for hydrostatic-pressure-dependent polymers proposed by one of the present authors. The yield surface is expressed by the first and second invariants of stress to describe the hydrostatic-pressure dependence. A plastic potential that is different from the yield surface is employed to describe the incompressibility of polymeric materials. Isotropic hardening is assumed. The proposed constitutive equations are implemented in the finite element code MSC.Marc with user subroutines. The calculated load-stroke curves appropriately describe the effect of introducing the hydrostatic-pressure dependence of PP. Moreover, the calculated results agree with the experimental ones for various thicknesses of specimens. Finally, the calculated distributions of bending stress and bending strain in the specimen also show the effects of hydrostatic-pressure dependence. [doi:10.2320/matertrans.P-MRA2007878]

(Received March 29, 2006; Accepted July 3, 2007; Published September 25, 2007)

Keywords: V-bending, finite element method, constitutive equation, hydrostatic-pressure-dependent plasticity, polypropylene, load-stroke curve, user subroutines

\section{Introduction}

Recently, a great many parts and components made of polymers have been used in, for example, automobiles, cellular phones, electric appliances, and office automation appliances. For the structural analysis of these parts, the static finite element method using the $J_{2}$ flow rule and von Misestype yield surface have mainly been performed. ${ }^{1-3)}$

It has been reported that polymer has a significant strain rate dependence and a hydrostatic-pressure dependence. ${ }^{4)}$ As a first approximation, however, the strain-rate dependence can be negligible in the structural analyses if the distribution of the strain rate of the deformed part is regarded as being almost uniform. On the other hand, the consideration of the hydrostatic-pressure dependence of polymer is important to achieve more precise estimation of the stress distribution of the polymer part subject to deformation.

One of the present authors has developed a timeindependent plastic constitutive equation that properly describes the hydrostatic-pressure dependence, from the abovementioned standpoint. The effect and limitation of the proposed equation have been verified by calculating the uniaxial loading behavior of a polymer that has a significant hydrostatic-pressure dependence, such as polypropylene (PP). ${ }^{5-7)}$

In the present study, the V-bending of PP is analyzed in order to verify the effect of using the hydrostatic-pressuredependent plastic constitutive equation for the structural or processing analyses by the static finite element method. The hydrostatic-pressure-dependent constitutive equation used in

*This Paper was Originally Published in Japanese in J. Jpn. Soc. Technol. Plasticity, 46-531 (2005), 332-336. the present paper has been developed in the framework of the isotropic hardening rule, the modified von Mises-type yield surface and the non associate flow rule. ${ }^{5,6)}$

The equation is implemented into the commercial finite element code MSC.Marc employing user subroutines. The effect of the hydrostatic-pressure dependence on the calculations is discussed. Furthermore, calculated results are compared with the corresponding experiments of V-bending performed by the present authors.

\section{Elastic-Plastic Constitutive Equation of Hydrostatic- Pressure-Dependent Polymer}

Let us summarize the elastic-plastic constitutive equation of the hydrostatic-pressure-dependent polymer used in the present study. 5,6 )

The total strain tensor can be divided into elastic and plastic parts as

$$
\varepsilon_{i j}=\varepsilon_{i j}^{e}+\varepsilon_{i j}^{p} .
$$

The elastic deformation of the polymer is assumed to be isotropic linear elastic and is given as

$$
\varepsilon_{i j}^{e}=\frac{1+v}{E} \sigma_{i j}-\frac{v}{E} \sigma_{k k} \delta_{i j},
$$

where $\delta_{i j}$ is Kronecker's delta, and $E$ and $v$ are Young's modulus and Poisson's ratio, respectively.

The hydrostatic-dependent yield surface is given as

$$
\left.\begin{array}{l}
f=(1-\beta) \sigma_{e q}+\beta I_{1}-\kappa=0 \\
I_{1}=\sigma_{k k}, \quad \sigma_{e q}=\sqrt{3 J_{2}}=\sqrt{\frac{3}{2} s_{i j} s_{i j}}
\end{array}\right\},
$$

where $I_{1}$ and $J_{2}$ are the first invariant of stress tensor and the 
second invariant of deviatoric stress tensor, respectively, and $\sigma_{e q}$ is the equivalent stress. Furthermore, $\kappa$ represents the isotropic strain hardening as a function of the equivalent plastic strain $\bar{\varepsilon}^{p}$.

Moreover, the coefficient $\beta$ is introduced to express the extent of the hydrostatic pressure dependence. If $\beta=0$, no hydrostatic pressure dependence is considered.

Although the hydrostatic pressure dependence is significant, most polymers have been reported to exibit incompressibility. Therefore, in order to take the incompressibility into account in the plastic constitutive equation, a plastic potential $g$ is introduced as

$$
g=\sqrt{3 J_{2}}
$$

According to the normality rule with respect to the potential $g$, a plastic constitutive equation is given as

$$
\dot{\varepsilon}_{i j}^{p}=\dot{\lambda} \frac{\partial g}{\partial \sigma_{i j}},
$$

where $\dot{\lambda}$ is a positive scalar function obtained using the consistency condition of the yield surface $\dot{f}=0$.

Equation (5) can be rewritten using eqs. (3) and (4) as

$$
\begin{aligned}
& \dot{\varepsilon}_{i j}^{p}=\frac{1}{H}\left[(1-\beta) \frac{3 s_{k l}}{2 \sqrt{3 J_{2}}}+\beta \delta_{k l}\right] \dot{\sigma}_{k l} \frac{3 s_{i j}}{2 \sqrt{3 J_{2}}} \\
& H=\frac{d \kappa}{d \bar{\varepsilon}^{p}}, \quad \bar{\varepsilon}^{p}=\int_{0}^{t}\left(\frac{2}{3} \dot{\varepsilon}_{i j}^{p} \dot{\varepsilon}_{i j}^{p}\right) d t
\end{aligned}
$$

For evolution equations of $\kappa\left(\bar{\varepsilon}^{p}\right)$, Swift's law and an exponential function are employed so that the actual plastic behavior can be properly expressed as

$$
\begin{aligned}
& \kappa=F\left(b+\bar{\varepsilon}^{p}\right)^{n}, \\
& \kappa=\sigma_{Y}+\left(\sigma_{Y}^{*}-\sigma_{Y}\right)\left[1-\exp \left(-c \bar{\varepsilon}^{p}\right)\right],
\end{aligned}
$$

where $F, b, n, \sigma_{Y}, \sigma_{Y}^{*}$ and $c$ are material constants.

\section{Stress Rate-Strain Rate Relation of Hydrostatic- Pressure-Dependent Polymer}

The proposed equations are to be implemented into the commercial finite element code MSC.Marc employing the user subroutine HYPELA for calculations of the V-bending of the polymer.

The stress rate-strain rate relation is given as

$$
\begin{aligned}
\dot{\sigma}_{i j} & =C_{i j k l}^{e p} \dot{\varepsilon}_{k l}, \\
C_{i j k l}^{e p} & =C_{i j k l}^{e}
\end{aligned}
$$

$$
-\alpha \frac{C_{i j p q}^{e} s_{p q}\left\{(1-\beta) s_{m n} C_{m n k l}^{e}+\frac{2}{3} \beta \sigma_{e q} \delta_{m n} C_{m n k l}^{e}\right\}}{\frac{4}{9} \sigma_{e q}^{2} H+(1-\beta) C_{i j k l}^{e} s_{i j} s_{k l}},
$$

$$
\alpha=\left\{\begin{array}{ll}
1, & \text { if } f=0 \text { and } \frac{\partial g}{\partial \sigma_{i j}} \dot{\sigma}_{i j} \geq 0 \\
0, & \text { if } f<0 \text { or } \frac{\partial g}{\partial \sigma_{i j}} \dot{\sigma}_{i j}<0
\end{array} .\right.
$$

For the plane-stress problem, Eqs. (8a)-(8c) can be rewritten, using the Voigt notation, as

$$
\left.\begin{array}{rl}
\left\{\begin{array}{c}
\dot{\sigma}_{x} \\
\dot{\sigma}_{y} \\
\dot{\tau}_{x y}
\end{array}\right\}=\left[\frac{E}{1-v^{2}}\left(\begin{array}{ccc}
1 & v & 0 \\
v & 1 & 0 \\
0 & 0 & \frac{1-v}{2}
\end{array}\right)-\alpha \frac{1}{S_{H}}\left(\begin{array}{ccc}
S_{x} \Sigma_{x} & S_{x} \Sigma_{y} & (1-\beta) S_{x} S_{x y} \\
S_{y} \Sigma_{x} & S_{y} \Sigma_{y} & (1-\beta) S_{y} S_{x y} \\
S_{x y} \Sigma_{x} & S_{x y} \Sigma_{y} & S_{x y}^{2}
\end{array}\right)\right]\left\{\begin{array}{c}
\dot{\varepsilon}_{x} \\
\dot{\varepsilon}_{y} \\
\dot{\gamma}_{x y}
\end{array}\right\}, \\
\sigma_{e q} & =\sqrt{\sigma_{x}^{2}-\sigma_{x} \sigma_{y}+\sigma_{y}^{2}+3 \tau_{x y}^{2}}, \\
S_{x} & =\frac{E}{1-v^{2}}\left(s_{x}+v s_{y}\right), \quad S_{y}=\frac{E}{1-v^{2}}\left(v s_{x}+s_{y}\right), \\
S_{x y} & =\frac{E}{1+v} \tau_{x y}, \quad S_{e q}=\frac{2}{3} \frac{E}{1-2 v} \sigma_{e q}, \\
S_{H} & =\frac{4}{9} \sigma_{e q}^{2} H+(1-\beta)\left(S_{x} s_{x}+S_{y} s_{y}+2 S_{x y} \tau_{x y}\right) \\
\Sigma_{x} & =(1-\beta) S_{x}+\beta S_{e q}, \quad \Sigma_{y}=(1-\beta) S_{y}+\beta S_{e q}
\end{array}\right\} .
$$

For the plane strain problem, Equations (8a)-(8c) can be rewritten as

$$
\begin{aligned}
& \dot{\sigma}_{z}=\eta\left(\dot{\sigma}_{x}+\dot{\sigma}_{y}\right) \\
& \eta=\left(v \sigma_{e q}+\frac{1}{2} E \overline{\varepsilon^{p}}\right) /\left(\sigma_{e q}+E \overline{\varepsilon^{p}}\right) \\
& \left.\begin{array}{l}
\sigma_{e q}=\left\{\left(\eta^{2}-\eta+1\right) \sigma_{x}^{2}+\left(2 \eta^{2}-2 \eta-1\right) \sigma_{x} \sigma_{y}\right. \\
\left.+\left(\eta^{2}-\eta+1\right) \sigma_{y}^{2}+3 \tau_{x y}^{2}\right\}^{1 / 2}
\end{array}\right\}, \\
& \left\{\begin{array}{c}
\dot{\sigma}_{x} \\
\dot{\sigma}_{y} \\
\dot{\tau}_{x y}
\end{array}\right\}=\left[\frac{E}{(1+v)(1-2 v)}\left(\begin{array}{ccc}
1-v & v & 0 \\
v & 1-v & 0 \\
0 & 0 & \frac{1-2 v}{2}
\end{array}\right)-\alpha \frac{1}{S_{H}}\left(\begin{array}{ccc}
S_{x} \Sigma_{x} & S_{x} \Sigma_{y} & (1-\beta) S_{x} S_{x y} \\
S_{y} \Sigma_{x} & S_{y} \Sigma_{y} & (1-\beta) S_{y} S_{x y} \\
S_{x y} \Sigma_{x} & S_{x y} \Sigma_{y} & S_{x y}^{2}
\end{array}\right)\right]\left\{\begin{array}{c}
\dot{\varepsilon}_{x} \\
\dot{\varepsilon}_{y} \\
\dot{\gamma}_{x y}
\end{array}\right\},
\end{aligned}
$$




$$
\left.\begin{array}{l}
S_{H}=\frac{4}{9} \sigma_{e q}^{2}\left\{H+\frac{3}{2}(1-\beta) \frac{E}{1+v}\right\}, \\
S_{e q}=\frac{2}{3} \frac{E}{(1+v)(1-2 v)} \sigma_{e q}, \\
S_{x}=\frac{E}{1+v} S_{x}, \quad S_{y}=\frac{E}{1+v} s_{y}, \quad S_{x y}=\frac{E}{1+v} \tau_{x y}, \\
\Sigma_{x}=(1-\beta) S_{x}+\beta S_{e q}, \quad \Sigma_{y}=(1-\beta) S_{y}+\beta S_{e q}
\end{array}\right\}
$$

Note that the elastic-plastic stiffness tensor (8b) and tangent stiffness matrices (9a) and (10a) are asymmetric, because the non associate flow rule, in which the yield surface $f$ is different from the plastic potential $g$, is employed.

\section{Experiments and Finite Element Analyses of V- bending of PP}

\subsection{Experimental conditions and apparatus}

In the present experiments, specimens were plates of PP (GrandPolypro J106W) that had been prepared by milling from extruded round bars. The width and length were $b=$ $15 \mathrm{~mm}$ and $l=95 \mathrm{~mm}$, respectively. The specimens had three different thicknesses of $t=4,5$ and $6 \mathrm{~mm}$, in order to examine the effect of the thickness on the bending behavior.

Figure 1 shows the experimental apparatus and the geometries of the punch and die used. The tip radius of the punch was $R_{p}=9 \mathrm{~mm}$, the bottom radius of the die was $R_{D}=$ $10 \mathrm{~mm}$, the interval between the die shoulder was $2 L=70$ $\mathrm{mm}$, and the radius of the die shoulder was $r_{D}=1 \mathrm{~mm}$. The punch speed was set to $v_{p}=0.5 \mathrm{~mm} / \mathrm{min}$. This slow speed was selected to avoid the effect of strain rate during the bending deformation of the specimens.

Plenty of mechanical oil was supplied to the specimens and tools to avoid the effect of friction.

\subsection{Analytical conditions}

Figure 2 shows a discretized model of the finite element analysis. Only the right side of whole specimen was modeled because of symmetry. The elements used were 4-node quadrilateral isoparametric elements. The numbers of elements and nodes were 446 and 522. The punch and die were modeled as rigid bodies.

Friction between the specimen and tools was neglected by adopting Coulomb friction coefficient $\mu=0$.

The material constants in the constitutive equation were determined so that the equation can properly express the experimental results of the uniaxial tension and compression, and were given as

$$
\begin{aligned}
& E=1.97 \mathrm{GPa}, \quad v=0.36 \\
& F=67.2 \mathrm{MPa}, \quad b=6.53 \times 10^{-4}, \quad n=0.217 \\
& \left.\begin{array}{l}
\sigma_{Y}=14.0 \mathrm{MPa}, \quad \sigma_{Y}^{*}=35.7 \mathrm{MPa}, \quad c=60.0 \\
\beta=0.17
\end{array}\right\},
\end{aligned}
$$

so that the results of uniaxial tensile and compressive tests were expressed properly.

Figure 3 shows the experimental and calculated results of the uniaxial tensile and compressive tests of PP. The ordinate and abscissa show the absolute values of uniaxial stress and strain, respectively. It is found that the proposed constitutive

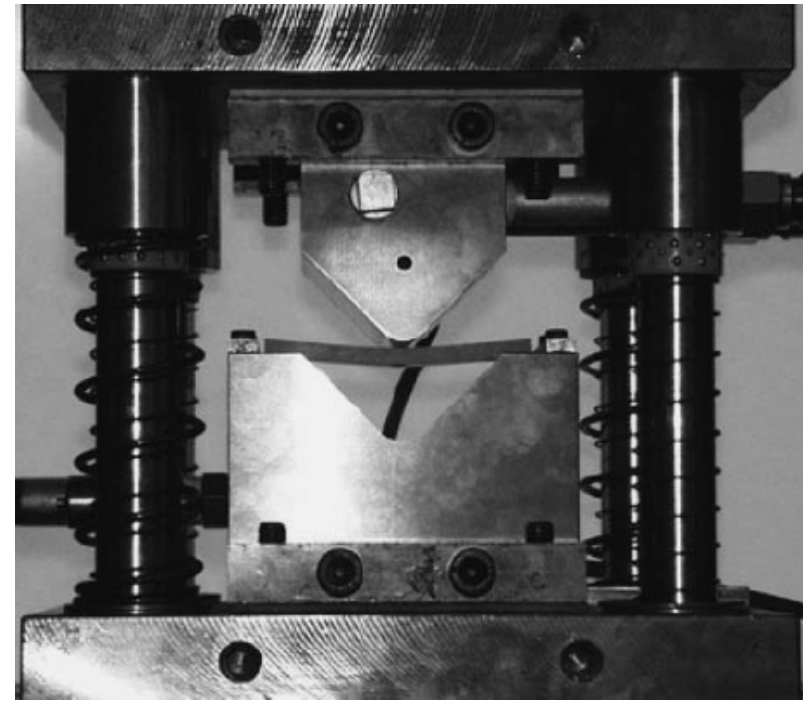

(a)

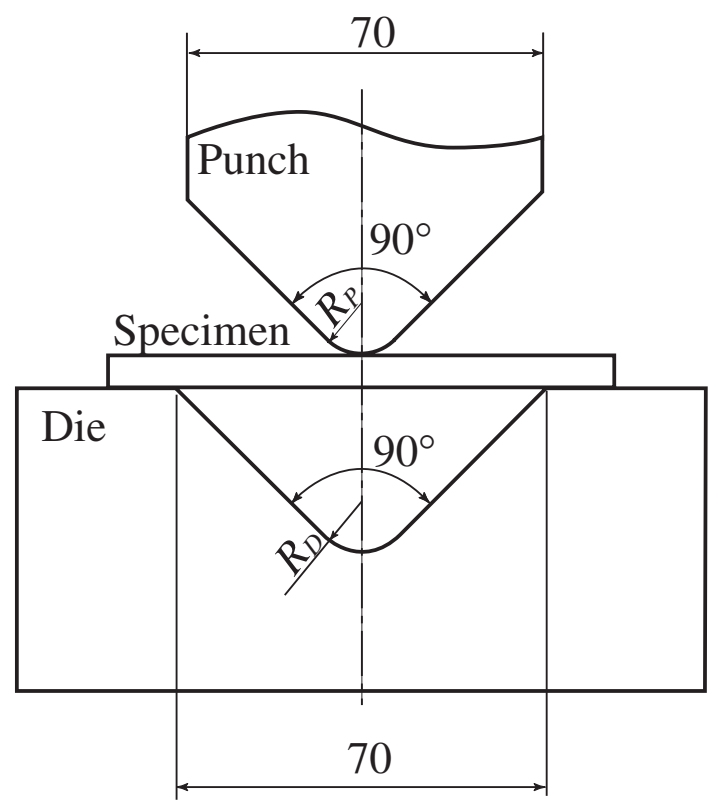

(b)

Fig. 1 V-bending apparatus and geometry of punch and die (a) V-bending apparatus (b) geometries of punch and die.

equation can accurately predict the difference between tensile and compressive stresses. The stresses estimated using Swift's law (7a) are higher than those estimated using exponential function (7b) in the range of large strain.

\subsection{Calculated results and discussion on V-bending}

Figure 4 shows the influence of Swift's law (7a) and 


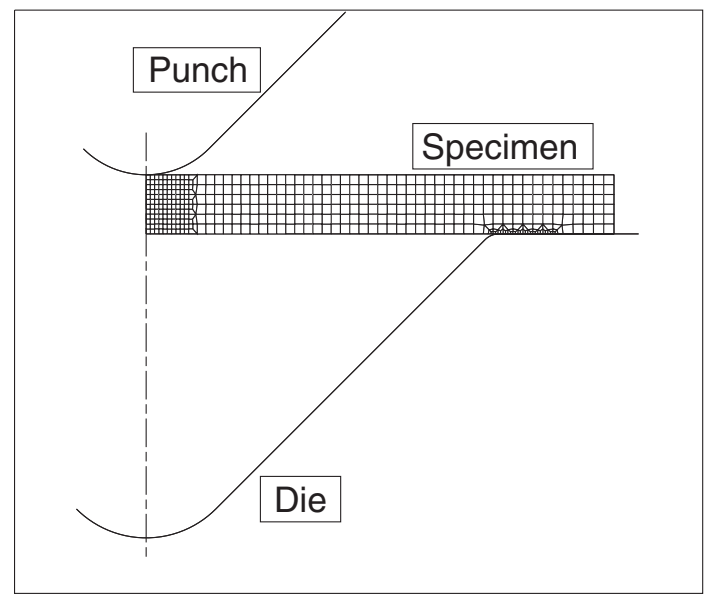

Fig. 2 Discretization of model analyzed.

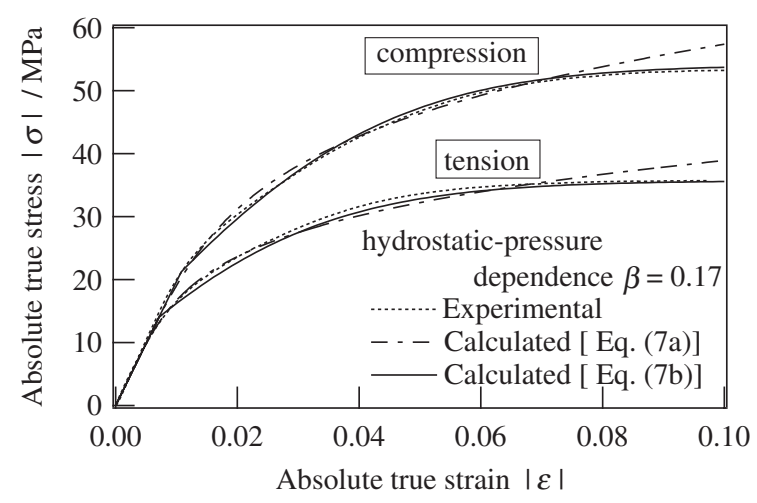

Fig. 3 Identification of material constants under uniaxial tension and compression.

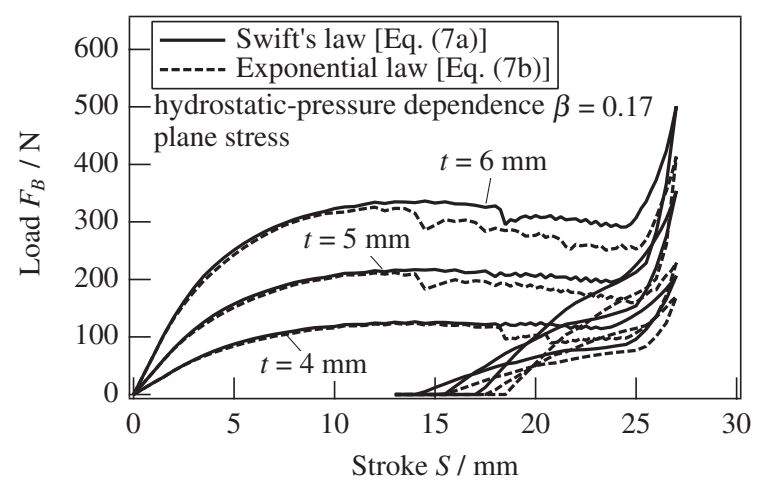

Fig. 4 Load-stroke curves for V-bending under plane stress calculated using Eqs. (7a) and (7b) with hydrostatic-pressure dependence $\beta=0.17$.

exponential function (7b) on load $F_{B}$-stroke $S$ curves under the plane-stress state. Solid and dashed lines are the results obtained using eqs. (7a) and (7b), respectively. Both equations give similar $F_{B}$ before reaching the maximal value. After the maximal value, $F_{B}$ obtained with eq. (7a) is more stable and less scattered than that obtained using eq. (7b). This is because the stress increment in eq. (7b) becomes almost zero as the strain increases. By considering the result of Fig. 4, Equation (7a) was employed in the calculations hereafter in the present study.

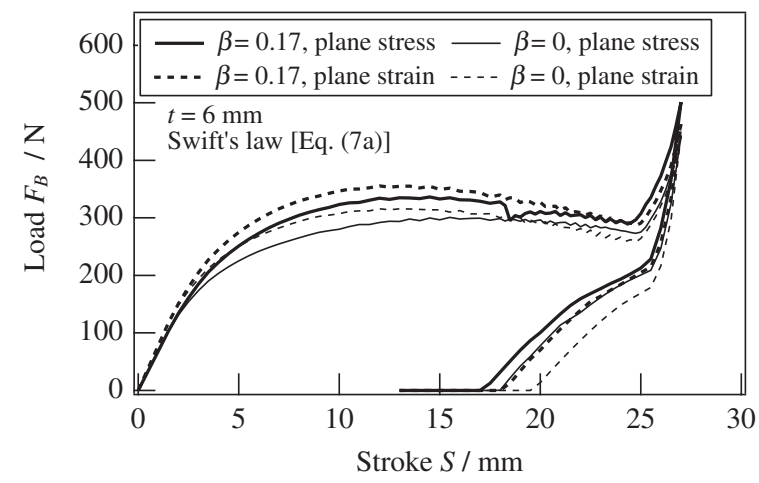

Fig. 5 Load-stroke curves for V-bending under plane stress and plane strain with hydrostatic-pressure dependence $\beta=0.17$ and 0 .

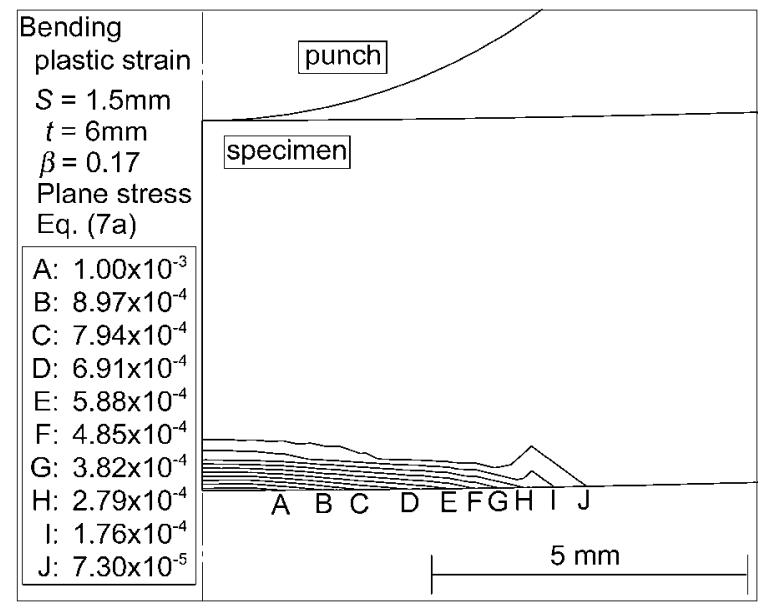

Fig. 6 Distribution of plastic bending strain for V-bending at stroke $S=1.5 \mathrm{~mm}$ under plane stress.

Figure 5 shows the $F_{B}-S$ curves in the case of $t=6 \mathrm{~mm}$. The results without the effect of hydrostatic pressure $(\beta=0)$ are also shown for reference. Regardless of $\beta$, results obtained under the plane-strain state yield higher estimates of load than under the plane stress. This is because the deformation in the direction of width is constrained in the case of the plane-strain state.

For the effect of hydrostatic pressure, $F_{B}$ when $\beta=0.17$ is larger than that when $\beta=0$. From the results, it can be concluded that the conventional von Mises-type yield surface essentially yields a value of $F_{B}$ smaller than the actual value.

The plane-stress state is used hereafter, because this state yields the better approximation in the range of the ratio of width to thickness of the specimen used in the present study, $b / t=2.5-3.75$. $^{\text {) }}$

Figure 6 shows the distribution of the bending plastic strain when $S=1.5 \mathrm{~mm}$, with the effect of the hydrostaticpressure dependence taken into account. Tensile bending plastic strain is observed on the tensile side of the bent specimen, whereas no compressive bending plastic strain is observed on the compressive side. This is because of the consideration of the effect of hydrostatic-pressure dependence. As observed in Fig. 3, the tensile yield stress is less than that of compression because of the hydrostatic-pressure dependence. Therefore, yielding on the tensile side takes place in advance of that on the compressive side. 


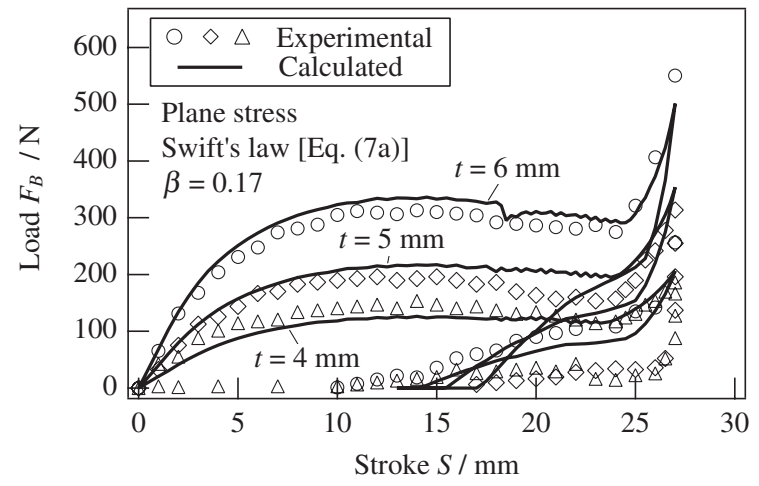

Fig. 7 Calculated and experimental results of load-stroke curves for Vbending of various thicknesses.

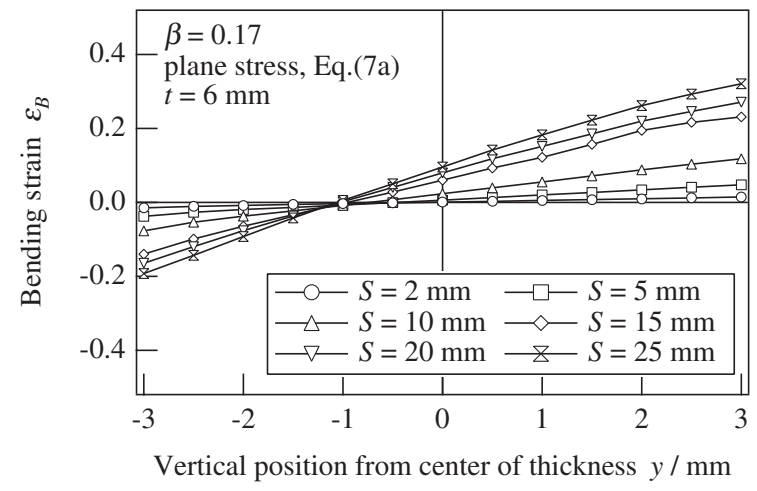

(a)

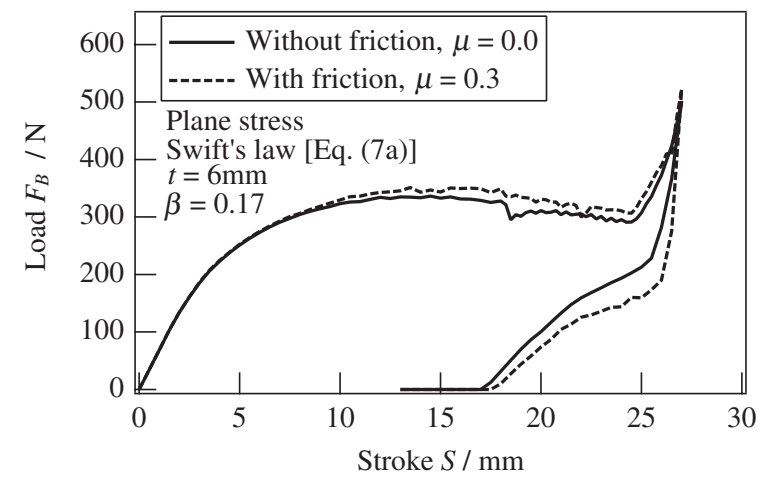

Fig. 8 Effect of friction coefficient on load-stroke curve for V-bending.

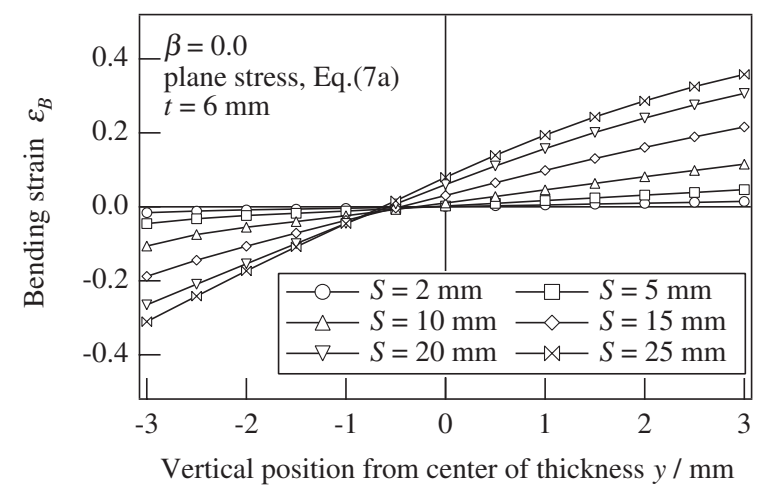

(b)

Fig. 9 Effect of hydrostatic-pressure dependence on bending strain distribution at each stroke (a) With hydrostatic-pressure dependence $\beta=0.17$ (b) Without hydrostatic-pressure dependence $\beta=0$.

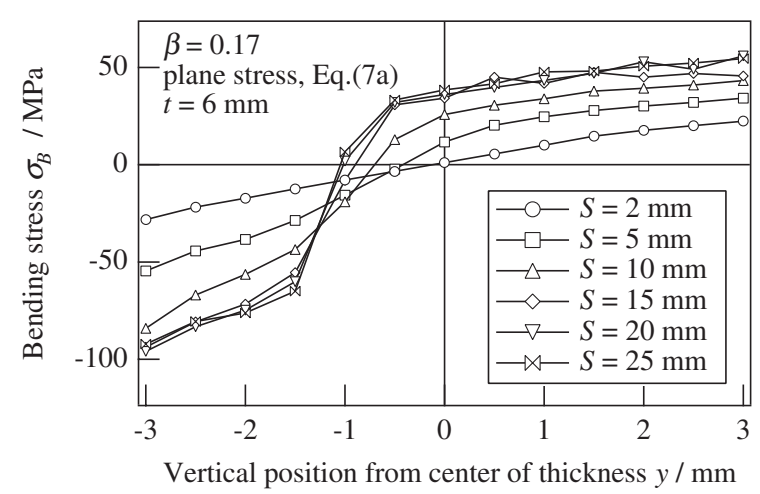

(a)

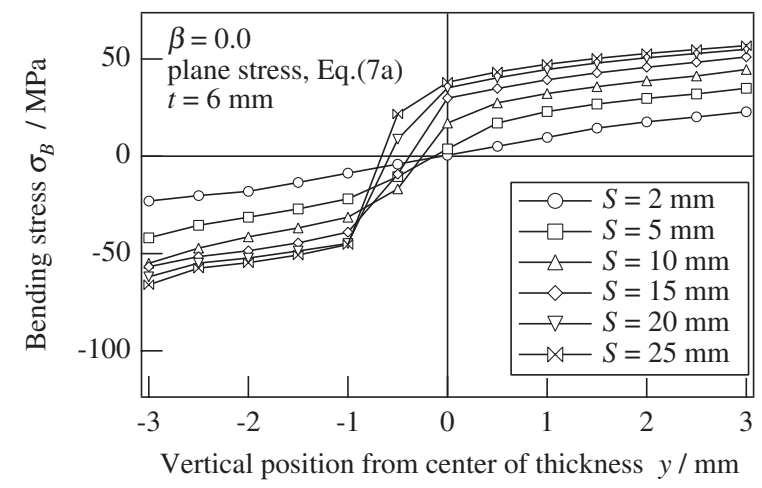

(b)

Fig. 10 Effect of hydrostatic-pressure dependence on bending strain distribution at each stroke (a) With hydrostatic-pressure dependence $\beta=0.17$ (b) Without hydrostatic-pressure dependence $\beta=0$.

Figure 7 shows the experimental and calculated $F_{B}-S$ relations for $t=4,5$ and $6 \mathrm{~mm}$. The solid lines and symbols show the calculated and experimental results, respectively. Good accordance between the experiments and calculations during the loading process is observed. On the other hand, the behavior is not expressed properly during the unloading process. This is because the simple isotropic hardening rule is employed in the present constitutive equation. To achieve a more precise description, constitutive equations that can predict the complicated hardening and time-dependent behaviors will be necessary. ${ }^{9,10)}$
Figure 8 shows the effect of friction on the $F_{B}-S$ relation. The solid and dashed lines are results calculated without friction $(\mu=0.0)$ and with friction $(\mu=0.3)$, respectively. It is observed that $F_{B}$ increases because of the existence of friction. However, the effect of friction is less significant in $\mathrm{V}$-bending than in other processes such as upsetting. Therefore, the assumption of no friction seems reasonable in the present study.

\subsection{Distribution of bending stress and strain}

Figures 9 and 10 show the effects of hydrostatic-pressure 
dependence on the distributions of bending stress and strain, respectively in the specimen. These calculations were carried out under the plane-stress state and with the thickness of $t=6 \mathrm{~mm}$. The abscissa of these figures shows the vertical position from the center of the thickness and is denoted by $y$.

In Fig. 9, the distribution of the bending strain is almost linear, namely, elastic, when $S$ is small. The neutral axis corresponds to the center of the thickness $(y=0)$. As $S$ increases, the distributions become nonlinear, or plastic. Furthermore, the neutral axis moves to the compression side of the bent specimen. This is because the width of the specimen on the compression side becomes larger than that on the tensile side due to the incompressibility of PP. The movement of the axis becomes greater when the effect of hydrostatic-pressure dependence is taken into consideration.

The effects of $\beta$ are observed significantly in case of the bending stress shown in Fig. 10. The compressive stress in $\beta=0.17$ is much larger than that in $\beta=0$.

It is confirmed that the PP characteristic of the compressive strength being higher than the tensile one can be expressed properly by considering the effect of hydrostatic-pressure dependence.

Note that the proposed constitutive equations and calculated results can also be verified by measuring the geometry of the cross section of the deformed specimen.

\section{Conclusion}

Finite element analyses of the V-bending behavior of hydrostatic-pressure-dependent PP were carried out using the hydrostatic-pressure-dependent plastic constitutive equation proposed by one of the present authors. As a result, the loadstroke curves and the distributions of bending stress and bending strain were revealed to be considerably influenced by the hydrostatic-pressure dependence. It is found that more precise analysis of parts or components made of polymer can be realized by using the constitutive equations that can properly describe the effect of hydrostatic pressure.

\section{Acknowledgement}

A part of the present paper is the result of the activity of the Group of Development of Constitutive Equation and Implementation to General-Purpose Finite Element Code. The authors would like to acknowledge the Simulation-Integrated-System Committee of the Japan Society of Technology of Plasticity for the financial support of the group.

\section{REFERENCES}

1) Y. Sugimoto, S. Otonari, H. Itou and H. Tomizawa: SAE Technical Papers, (1998), 980108.

2) T. Takahara, M. Mikami, J. Chen and Y. Sugimoto: SAE Technical Papers, (2001), 2001-01-3084.

3) M. Todo, K. Arakawa and K. Takahashi: Key Engng. Mat. 183-187 (2000) 409-414.

4) I. M. Ward and J. Sweeney: An Introduction to the Mechanical Properties of Solid Polymers, 2nd Ed., (John Wiley \& Sons, 2004).

5) Y. Sanomura: Mat. Sci. Res. Int. 9 (2003) 243-247.

6) Y. Sanomura: J. Soc. Mat. Sci. Japan 50 (2001) 968-972.

7) Y. Sanomura and K. Hayakwa: J. Soc. Mat. Sci. Jpn 53 (2004) 143-149 (in Japanese).

8) K. Kazama and Y. Nagai: J. Jpn. Soc. Technol. Plasticity 45 (2004) 4044 (in Japanese).

9) M. Mizuno and Y. Sanomura: Proc. ANTEC 2004, (2004), 1912-1916.

10) Y. Sanomura and M. Mizuno: J. Jpn. Soc. Strength Fract. Mat. 38 (2004) 7-13 (in Japanese). 